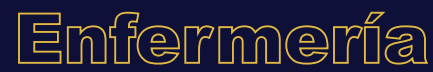

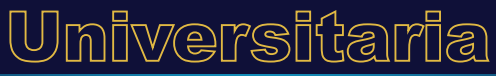

\section{Análisis y autoevaluación de las competencias tutoriales en un programa de posgrado en enfermería}

\author{
Analysis and self-assessment of the tutorial \\ competences in a nursing graduate program
}

\section{Análise e autoavaliação das competências tutoriais em um programa de pós-graduação em enfermagem}

\section{Piña-Jiménez ${ }^{\mathrm{a}, *}, \mathrm{G}$. Ponce-Gómez ${ }^{\mathrm{b}}$}

ORCID

a $0000-0001-5721-7268$

b $0000-0002-1532-8207$

Universidad Nacional Autónoma de México, Escuela Nacional de Enfermería y Obstetricia, Ciudad de México, México

Recibido: 15 octubre 2019

Aceptado: 27 enero 2020

RESUMEN

Introducción: El tutor es fundamental en la formación de estudiantes del posgrado, su trabajo no se enfoca únicamente en la dirección de la tesis; además debe fomentar el desarrollo de otras habilidades en el alumno al incorporarlo al conocimiento tácito en comunidades de investigación. Esto implica que el tutor muestre competencias en la tutoría, de ahí la importancia de reconocerlas y autoevaluarlas tanto en forma crítica como reflexiva para su mejora. 
Objetivo: Analizar y autoevaluar las competencias de los tutores de un posgrado en enfermería.

Método: Los tutores fueron invitados a participar de manera libre y voluntaria en grupos focales, con el propósito de analizar, discutir y autoevaluar sus competencias; se empleó una rúbrica como elemento detonador, que describe cualitativamente diez competencias que deben tener los tutores de posgrado, en cuatro niveles de dominio.

Resultados: La discusión sostenida al interior de los grupos focales fue documentada para su codificación y análisis, se obtuvieron categorías que aludieron a: 1) aspectos éticos y el respeto a las autorías de los estudiantes en publicaciones; 2) la relevancia de la transferencia del conocimiento a problemas específicos del campo de enfermería; 3) la relación tutoralumno destacando vínculos de menor y mayor dependencia.

Conclusiones: Se identificó el reconocimiento de las competencias que requieren los tutores en este nivel, se generó una autoevaluación crítica y reflexiva, sobre lo que se ha realizado en la práctica educativa. Los tutores ubicaron sus niveles de competencia en desarrollo y consumado.

Palabras clave: Tutoría; autoevaluación; educación de postgrado en enfermería; enseñanza; competencias docentes; México.

\begin{abstract}
Introduction: Tutors are fundamental in the formation of graduate students and their work is not only focused on the direction of the students' theses. Tutors also encourage the development of other skills, including the integration of students into research communities. This implies that tutors must demonstrate tutoring competencies; and therefore, it is important to recognize and assess these competencies from critical and reflexive points of view.

Objective: To analyze and self-assess the competencies of tutors in a nursing graduate program.

Method: Tutors were invited to participate in a free and voluntary manner in diverse focal groups with the aim of analyzing, discussing, and self-assessing their own competencies. Ten competencies in four domain levels were qualitatively assessed.

Results: The discussion within the focal groups were documented and coded, and the following categories were identified: 1) ethical aspects and the respect of the authorship of students in publications; 2) the relevance of the transference of knowledge to specific problems in the field of nursing; 3 ) the tutor-student relationship highlighting the different levels of dependence associations.

Conclusions: The identification of the competencies which tutors need to demonstrate at this curricular level was acknowledged. A critical and reflexive self-evaluation regarding the education practice was generated. Tutors identified their levels of competency.

Keywords: Mentoring; self-assessment; education, nursing, graduate; teaching; teaching competencies; Mexico.
\end{abstract}

\title{
RESUMO
}

Introdução: Otutor éfundamental na formação de estudantes de pós-graduação, seutrabalho não se concentra unicamente na direção da tese; aliás deve fomentar o desenvolvimento de 
outras habilidades no aluno incorporando-o ao conhecimento tácito em comunidades de pesquisa. Isso implica que o tutor mostre competências na tutoria, daí a importância de reconhecê-las e auto avaliá-las de forma crítica e reflexiva para seu aprimoramento.

Objetivo: Analisar e autoavaliar as competências dos tutores de uma pós-graduação em enfermagem.

Método: Os tutores foram convidados para participar de maneira livre e voluntária em grupos focais, com o propósito de analisar, discutir e autoavaliar suas competências; foi utilizada uma rubrica como elemento desencadeador, que descreve qualitativamente dez competências que os tutores de pós-graduação devem ter, em quatro níveis de domínio.

Resultados: A discussão realizada no interior dos grupos focais foi documentada para sua codificação e análise, obtiveram-se categorias que aludiram a: 1) aspectos éticos ao respeito da autoria dos estudantes em publicações; 2) a relevância da transferência do conhecimento para problemas específicos do campo de enfermagem; 3) a relação tutoraluno evidenciando vínculos de menor e maior dependência.

Conclusões: Identificou-se o reconhecimento das competências que os tutores requerem neste nível, gerou-se uma autoavaliação crítica e reflexiva, sobre o que tem sido realizado na prática educativa. Os tutores localizaram seus níveis de competência em desenvolvimento e alcançado.

Palavras chave: Tutoria; autoavaliação; educação de pós-graduação em enfermagem; ensino; competências docentes; México.

\section{INTRODUCCIÓN}

Las transformaciones que demanda la educación en el posgrado permiten centrar nuestra atención en el trabajo que desarrolla el tutor, como un elemento decisivo en la formación de las nuevas generaciones. Por tal razón, es importante tener una concepción clara de lo que es la tutoría y la consideración de la complejidad que ésta comprende en la práctica educativa, dadas las diversas situaciones que acompañan los trayectos de formación de los estudiantes y el sentido que adquieren las acciones que realiza el tutor en su interacción con el tutorado, quien considera tanto las características personales del estudiante como los propósitos de formación que institucionalmente se persiguen, además de las circunstancias que pueden acompañar a dicho proceso.

En la revisión de la literatura ${ }^{1}$, el concepto de tutoría en su origen nos remite a la función que realiza Méntor cuando Odiseo debe partir a la guerra, pidiéndole que se haga cargo de su hijo Telémaco bajo la figura de tutor y guía en su ausencia. Otras concepciones destacan la necesidad de esta figura para apoyar la formación integral de los jóvenes, como es el caso de las universidades anglosajonas², en el que se muestra un interés especial por favorecer el desarrollo personal del estudiante.

Otros autores ${ }^{3}$ refieren que sólo se puede acompañar a alguien si sabe a dónde se va, lo que sugiere que la persona acompañada, tiene libertad y asume una responsabilidad, es decir, el tutor podrá aconsejar o sugerir, pero el tutorado es quien ha de enfrentar y asumir la responsabilidad en el trayecto. Aspecto que tiene sentido cuando hablamos de una formación a nivel de posgrado, en el que se asigna a los alumnos un tutor, con el fin de recibir acompañamiento en su trayecto de formación, mostrándole los retos y las vías para alcanzar de mejor forma sus propósitos.

En la revisión analítica de la literatura que realizaron De la Cruz et al. ${ }^{1,4}$ se vislumbran las contribuciones y las tareas pendientes del corpus teórico que dan sustento a la tutoría en la educación 
superior. Entre los atributos del tutor se destacan aspectos formativos (dominio de conocimiento en un campo de estudio), didácticos (herramientas que facilitan el proceso enseñanza-aprendizaje), interpersonales (relación y empatía con los alumnos), cognoscitivos (habilidades para la organización del pensamiento) y éticos (honestidad y respeto ganado en el ámbito académico).

Los trabajos de Martínez-González et al..$^{5}$ exploraron inicialmente las competencias de los tutores de Posgrado en las diferentes áreas del conocimiento en una universidad pública, al interrogarlos sobre sus buenas prácticas como tutores; las competencias en la investigación y en la interacción tutor estudiante fueron las más destacadas. En la investigación de Difabio-de Anglat ${ }^{6}$, el análisis se centra en las funciones del tutor en el posgrado y se destaca la importancia de la realimentación del trabajo escrito en forma constructiva, mientras que González-Juárez, profundiza en el nivel de competencia que desarrollan los alumnos para realizar investigación durante su formación de posgrado, al poner de relieve la mediación del trabajo del tutor.

Varios autores coinciden en que el trabajo del tutor no se agota únicamente en la dirección del trabajo de tesis, pues es preciso incorporar a los alumnos al espacio de reflexión, producción y generación del conocimiento propio de la profesión; familiarizándolos tanto con los avances en el campo de estudio, como con las experiencias y prácticas de las comunidades académicas y redes de investigación, para conectarlo no sólo con las formas de producción del conocimiento sino también con el conocimiento tácito que poseen dichas comunidades ${ }^{8}, 9$.

Cardoso et al..$^{10}$ analizó las competencias de los docentes de tres posgrados del área de Administración, en el que identificaron los principales ejes que direccionan la actividad docente, los cuales fueron: el desarrollo de la investigación a través de proyectos y su aportación al área de conocimiento, así como la publicación de los resultados obtenidos en estos; es decir, la principal competencia que mostraron los tutores fue su capacidad para dirigir a sus alumnos, concluir en tiempo y forma el proyecto además de generar nuevas aportaciones al campo de estudio de la Administración.

Existe evidencia considerable de que el trabajo de los tutores ha sido medido y valorado en las instituciones educativas, por un lado, en función del número de alumnos graduados (lo que permite ver cuantitativamente un resultado concreto en cada generación) o bien a través de la opinión que emiten los estudiantes sobre éstos. Los resultados en las evaluaciones no siempre se utilizan con fines de retroalimentación y mejora para el trabajo del tutor, más bien se traducen en recomendaciones que destacan aquello que éste no cubre, lo que conlleva a identificar la evaluación en un sentido negativo o de control, que no aporta una transformación y mejora de la misma ${ }^{11,12}$.

\section{Visibilizar los elementos que comprende el trabajo del tutor.}

Las formas de evaluación, si bien generan datos, no visibilizan todos los elementos que entran en juego durante el proceso de formación, con los que los tutores deben contender, ni refieren cómo enfrentan los acelerados cambios que derivan de la sociedad del conocimiento, ni la presencia de una comunidad de alumnos de gran heterogeneidad cultural, socio económica y de habilidades para el estudio. Situación que demanda a los tutores el despliegue de diversas acciones, como son las comunicativas y motivacionales ${ }^{13}$ entre otras, ajustadas a las necesidades y requerimientos de cada estudiante.

Desde el contexto educativo institucional, se recomienda a los tutores acordar con los alumnos, las actividades que en forma conjunta van a realizar durante el trayecto de formación $n^{14}$, a manera de 
un contrato pedagógico, que contribuya a su formación integral, así como al desarrollo y conclusión exitosa de su trabajo para obtención del grado.

Si el trabajo de tutoría se vislumbra como un elemento que favorece la formación integral del alumno y promueve su independencia intelectual4,15, es pertinente que el tutor de posgrado reconozca de manera cualitativa las competencias que necesita desplegar en su desempeño como tutor y se vea implicado en un proceso de reflexión y análisis con sus pares, sobre su propia práctica educativa, de manera que pueda contar con mayores elementos para direccionarla.

\section{Concepto de competencia}

El concepto de competencia como lo ha definido Perrenoud ${ }^{16}$ alude a la capacidad aprendida para realizar una tarea, función o rol, que implica la movilización de recursos cognitivos para hacer frente a una situación. El autor destaca que la competencia si bien comprende conocimientos, habilidades, actitudes y valores, no se reduce a uno solo de estos elementos por separado, sino a la capacidad de su integración y movilización conjunta acorde a la situación específica que lo requiere.

El proceso de formación de competencias tutoriales tiene en sus raíces tanto la formación como la trayectoria profesional del tutor y de manera importante la experiencia en su práctica educativa, en su interacción con los alumnos. Un tutor experimentado tendrá mejores elementos para contender por un nivel de competencia mayor, porque ha atravesado por una gama amplia y diversa de situaciones, que le aportan una visión compleja de la tutoría. En el terreno del desarrollo de las competencias, el alcance de un nivel de dominio no es conclusivo, es decir, la persona puede ubicarse a sí misma en un nivel incipiente, lo que no significa que permanecerá allí por siempre, sino que eventualmente podrá avanzar y alcanzar mejores niveles de dominio, en razón de la experiencia y el afrontamiento resolutivo (en una gama amplia y diversa de situaciones), aspecto que ha sido estudiado por Denyer et al. ${ }^{17}$ al abordar la forma en que se alcanzan mayores niveles de dominio en las competencias.

En este sentido, nuestro escrito presenta algunos resultados que derivaron de una investigaciónacción, cuyo objetivo fue que los tutores que participan en un Programa de Posgrado en Enfermería de una universidad pública, reflexionen y autoevalúen sus competencias como tutores, a la luz de una rúbrica elaborada por De la Cruz et al. ${ }^{18}$ que define cualitativamente diez competencias de los tutores en el posgrado agrupadas en cuatro niveles de dominio: inicial, en desarrollo, consumado y ejemplar.

\section{Autoevaluación del tutor}

De manera conceptual las bondades de la autoevaluación son el favorecimiento de la autocrítica y el desarrollo de las personas, lo que propicia a su vez la formación continua ${ }^{19}$, la reflexión de la práctica en el oficio de enseñar y la transformación de esta, como lo han destacado Schön y Perrenoud ${ }^{20,21}$. La autoevaluación tiene una función formativa, se realiza en forma participativa, con el fin de generar un aprendizaje y la mejora continua ${ }^{22}$. Esto puede obtenerse si en los ejercicios de autoevaluación prevalece un clima adecuado de trabajo y se busca tanto la transformación como el crecimiento, en lugar de un señalamiento punitivo ${ }^{23}$.

\section{METODOLOGÍA}

El propósito de esta investigación es contribuir al mejoramiento de la práctica educativa de los tutores de un Posgrado de Enfermería, a través del análisis y evaluación de las competencias que 
estos presentan; el proyecto fue dictaminado por un Comité de Investigación de la entidad educativa universitaria respectiva, asignándole un registro. En la ejecución se respetaron los aspectos éticos y la rigurosidad científica de la investigación cualitativa en cuanto a credibilidad, transferibilidad y confirmabilidad.

El diseño se apegó a la modalidad de investigación-acción cualitativa, dado que esta metodología permite centrarse en la mejora de la práctica educativa de los tutores, a partir de un análisis crítico y reflexivo, de manera que los tutores como principales protagonistas del proceso educativo y en su relación con sus alumnos tutorados, tomen conciencia de su papel y sean partícipes de este proceso de transformación, como lo mencionan Carr y Kemmis² en su definición teórica de la investigaciónacción aplicada al profesorado.

\section{Conformación de grupos focales para análisis y autoevaluación}

La reflexión de las competencias de los tutores de posgrado en sus cuatro niveles de dominio, retoma la experiencia en la práctica educativa; en este documento abordamos la última fase de la investigación-acción, en la que se conformaron grupos focales con los tutores, quienes reconocieron y analizaron cada una de las competencias comprendidas en el instrumento y externaron sus puntos de vista respecto a las mismas.

A partir del reconocimiento y análisis de cada una de las competencias, se solicitó a los tutores que autoevaluaran su trabajo, con base en el instrumento proporcionado por las autoras. Esta fase buscó generar una mayor reflexión e involucramiento del tutor, con lo que realiza en su práctica educativa cotidiana, en un contexto horizontal de reflexión grupal con sus pares; como resultado se obtuvo un análisis y su propia autoevaluación.

Los grupos focales se conformaron con tutores activos del Programa de Posgrado en Enfermería, a quienes se les hizo una invitación abierta para participar.

Se integraron tres grupos con 5, 6 y 10 participantes ( $n=21$ tutores), lo que representó una participación del 50\% de la plantilla total de tutores en ese momento, de las tres entidades académicas que participan en el Programa.

Instrumento de análisis y autoevaluación: Se entregó a cada tutor un instrumento para su lectura y análisis denominado Rúbricas de competencias tutoriales, elaborado por De la Cruz et al. ${ }^{18}$. Este instrumento tiene el propósito de guiar, modelar y realimentar el desempeño de los tutores del posgrado; reúne diez competencias, agrupadas en: $a$. formativas socializadoras y $b$. interpersonales, cada una de ellas presenta los cuatro niveles de dominio: incipiente, en desarrollo, consumado y ejemplar (Tabla 1). Para dar idea al lector, incluimos el desglose amplio de los cuatro niveles de dominio únicamente para la competencia de socialización (Tabla 2).

Como ya se ha mencionado con anterioridad, el proceso de desarrollo de las competencias de los tutores depende de su exposición a una gama amplia y diversa de situaciones, esto puede ejemplificarse si consideramos que los alumnos de posgrado poseen diferentes estilos y maneras de aprender, de investigar y de integrarse al Programa de formación, lo que impulsa a los tutores experimentar formas diferenciadas de conducirse para alcanzar los objetivos formativos propuestos y esperados por el programa.

Una vez que el tutor leyó el instrumento se inició su discusión y análisis en forma grupal. Con el fin de favorecer la participación de todo el grupo, se procuró que la discusión de cada competencia se iniciara rotando la participación de los integrantes; al concluir la discusión en el grupo focal, se solicitó a cada tutor de manera individual, autoevaluar su nivel de dominio en cada una de las 
competencias, de acuerdo a las descripciones que comprende la rúbrica. Para realizar el proceso de análisis de los datos, se codificaron los contenidos de cada grupo focal, para posteriormente conformar las categorías emergentes.

Se revisaron las respuestas por grupo para después realizar la comparación entre estos con el fin de identificar puntos de coincidencia (los cuales se aborda en este trabajo) y de diferenciación. Las narrativas fueron clasificadas con la letra P (participante) seguido de un número del 1 al 21 según orden de registro y con una $G$ para el número de Grupo focal al que pertenecían.

\section{Tabla 1. Competencias tutoriales en el Posgrado}

\section{Competencias formativas socializadoras}

1. Formación en investigación: formar posgraduados capaces de realizar investigación original e independiente.

2. Docencia: guiar el proceso formativo de los estudiantes a fin de que logren una visión amplia del campo de conocimientos, así como su relación con otros campos disciplinares.

3. Formación profesional: desarrollar la capacidad de los alumnos para solucionar problemas en el contexto de práctica.

4. Consejería académica: asesorar al estudiante en los aspectos académico-administrativos del programa de posgrado.

5. Socialización: integrar a los estudiantes a las comunidades profesionales o de investigación en su campo.

6. Auspicio académico: favorecer que los estudiantes obtengan los recursos (humanos, materiales, infraestructura y financieros) suficientes para realizar sus proyectos de investigación.

7. Apoyo psicosocial: respaldar a los estudiantes para que tengan las condiciones sociales, culturales y emocionales indispensables para la obtención de sus metas.

\section{Competencias interpersonales}

8. Comportamiento ético: favorecer la autonomía y libertad de los estudiantes: Respetar autorías y no abusar de su estatus como tutores para beneficiarse de los alumnos.

9. Clima de interacción: propiciar la comunicación, confianza y empatía con los estudiantes.

10. Profesionalismo: mostrar compromiso y responsabilidad con la formación de los estudiantes.

Fuente De la Cruz-Flores G, Díaz-Barriga Arceo F, Abreu-Hernández LF. La labor tutorial en los estudios de posgrado: Rúbricas para guiar su desempeño y evaluación. Perf. educ. 2010; 32(130): 83-102.

https://doi.org/10.22201/iisue.24486167e.2010.130.20624

\section{RESULTADOS}

Las características de los 21 tutores que participaron en los grupos focales fueron las siguientes: predominó el sexo femenino (90\%) sobre el masculino; la edad promedio fue de 56.7 años; poco más del 70\% cuenta con doctorado; respecto a su antigüedad en el programa, el rango de mayor antigüedad fue de 11 a 15 años.

A partir de los hallazgos que emergieron en la discusión de los grupos focales se conformaron las siguientes categorías: comportamiento ético del tutor; la transición de la investigación descriptiva a la intervención controlada y la libertad del alumno en su relación con el tutor.

\section{El comportamiento ético del tutor}

Dentro de las 10 competencias descritas en la rúbrica, el análisis de la competencia comportamiento ético generó gran discusión en los tres grupos focales, los tutores expresaron que era de su conocimiento la existencia de diversas conductas no éticas que algunos tutores presentan, tales 
como no atender al alumno por viajar con frecuencia; no respetar la autoría y la colaboración del alumno en los documentos que publica el tutor, o bien exigir al estudiante a asistir y participar en un evento (foros, congresos, etc.) sin que él esté de acuerdo.

Ante dicha situación los tutores consideraron que se debe depurar [el padrón de tutores] y denunciar los casos, así como esclarecer los lineamientos respecto al tema de las autorías. Algunos comentarios fueron:

...informar a los tutores y alumnos sus derechos sobre la autoría... (р3 93).

...quien no tiene ética va a buscar sacar provecho del alumno... (p1 g1).

... tenemos dos grupos de alumnos... el que guío, lo acompaño y se beneficia...y

el alumno que es brillante y se va a eventos [por propia iniciativa], o se le

impone algo... eso es abuso de poder... (p3 g2). Se refiere a que el tutor le impone

asistir y presentar un trabajo en un evento, sin su consentimiento.

Tabla 2. Competencia 5 de socialización.

Descripción cualitativa de los cuatro niveles de ejecución

Niveles de
ejecución y puntaje

1. Incipiente - Los tutores se limitan a asesorar la tesis de grado, pero no integran al alumno a su grupo de investigación.

- Los alumnos interaccionan con el grupo de investigación del tutor sólo de manera eventual para alguna consulta técnica.

- No se propicia la interacción con otros grupos de investigación internos o externos a la institución.

2. En desarrollo $\quad$ Los tutores favorecen la integración parcial de sus alumnos a su grupo de investigación, asignándoles roles definidos de carácter técnico instrumental.

- No se propicia la interacción con otros grupos de investigación internos o externos a la institución.

3. Consumado - Los tutores favorecen la integración de los alumnos con su grupo de investigación, asignándoles roles definidos; conforme avanzan los impulsan a adoptar roles cada vez más relevantes.

- Propician sistemáticamente la interacción con otros grupos de investigación internos o externos a la institución, e impulsan a sus alumnos a participar en eventos académicos (congresos, simposios, etc.)

4. Ejemplar - Los tutores favorecen la integración de los alumnos a grupos de investigación, asignándoles roles definidos conforme avanzan los impulsan a adoptar roles cada vez más relevantes.

- Propician sistemáticamente la interacción con otros grupos de investigación internos o externos a la institución, e impulsan a sus alumnos a participar en eventos académicos (congresos, simposios, etc.)

- Los tutores propician que los alumnos colaboren con otros grupos de investigación nacionales y extranjeros.

- Estimulan que los alumnos amplíen y consoliden sus propias redes, así como se incorporen y asuman una posición de liderazgo en sociedades científicas nacionales e internacionales.

Fuente De la Cruz-Flores G, Díaz-Barriga Arceo F, Abreu-Hernández LF. La labor tutorial en los estudios de posgrado: Rúbricas para guiar su desempeño y evaluación. Perf. educ. 2010; 32(130): 83-102. https://doi.org/10.22201/ iisue.24486167e.2010.130.20624 (por razones de espacio sólo incluimos el desglose de una competencia en sus cuatro niveles de dominio, a fin de que el lector pueda apreciar las características descriptivas que ofrece el instrumento). 
Uno de los grupos focales consideró que la competencia ética a diferencia de las otras competencias, no es un atributo que se pueda ir perfeccionando gradualmente, sino que se tiene o no se tiene.

... se puede avanzar en forma progresiva en estas competencias, pero

los aspectos éticos o los tienes o no los tienes... (p5 93).

\section{De la investigación descriptiva a la intervención controlada}

La discusión de la competencia de formación en la investigación, se vincula con dar respuesta a problemáticas concretas para el cuidado de la salud de las personas, objeto de estudio de la enfermería.

En este sentido se externó una pregunta al tutor ...¿¿cómo hacer la vinculación con la práctica?..., se argumenta que ello requiere de otras habilidades por parte del tutor en el terreno de la intervención profesional de la enfermería, como respuesta tuvimos ...se requieren de otros actores que estén en la práctica, o bien iniciar trabajos en escenarios de práctica... (p5 g3), se dice que para transitar de la investigación meramente descriptiva a una investigación de intervenciones controladas, que aporten alternativas o soluciones a problemas específicos del cuidado de la salud, se requieren de otros escenarios y de otras habilidades del tutor-investigador. Esto último implicaría colocar a los alumnos en escenarios de práctica, en donde se vislumbren problemáticas que se analicen y demanden la formulación de alternativas de solución.

Por otro lado, los tutores advierten que en los estudiantes prevalece un interés por la obtención del grado versus interés por la investigación. Al abordar este aspecto se expusieron argumentos que destacan que los alumnos realizan los estudios de maestría con el propósito central de obtener una mejora en su condición laboral, es decir, lograr un ascenso a nivel escalafonario en la institución de salud en la que laboran.

... la tutoría en el Programa de Maestría en Enfermería, atiende solamente a tener un

documento, un grado, pero después de eso, jamás se vuelven a dedicar a la investigación... (p2 g3).

Esta afirmación puede indicar que los alumnos no adquieren un interés genuino por la investigación en el campo de estudio de la enfermería, ni por continuar con su trayectoria profesional en este terreno; se acercan a ella para generar su trabajo de tesis y obtener el grado. Sin embargo, este aspecto también puede llevarnos a considerar que los egresados de estudios de posgrado, si bien adquieren habilidades para el desarrollo de la investigación, no se están integrando (como se espera) a las actividades de investigación y generación del conocimiento en el campo de trabajo, por lo que el egresado termina incorporándose en un nivel operativo, donde sus elementos formativos en la investigación no son aprovechados.

Asimismo, los tutores hicieron la observación de que la rúbrica no diferencia los alcances en la formación del nivel maestría con el de doctorado y son programas con alcances diferentes en la producción de investigación y a la interdisciplinariedad, para generar propuestas de solución a problemas sociales de gran complejidad.

.... toda la tendencia es hacia la interdisciplinariedad, pero es dificil que se dé la interdisciplinariedad, pareciera que es lo ideal, pero no checa en nuestra realidad... (p3 g1). 
La incorporación de los alumnos a los grupos y comunidades de investigación, descrita en la competencia de socialización, requiere por un lado un desarrollo más sólido de las líneas en las que participan los tutores (a fin de que se consoliden en un área de experticia). Por otro lado, las instituciones de salud deben reorganizar a los equipos interdisciplinarios de investigación para que el estudiante se incorpore e identifique la importancia del trabajo colaborativo entre pares y con otros profesionales del área de la salud que participan en los problemas actuales de investigación, situación que beneficiará a ambas partes, es decir, el estudiante aplicará las habilidades aprendidas en investigación y las instituciones contarán con mayores recursos humano para desarrollarla.

\section{Libertad del alumno en su relación con el tutor}

Este aspecto derivó del análisis de las competencias interpersonales que aluden al clima de interacción y al profesionalismo del tutor, en que se espera el establecimiento de la comunicación, la confianza y la empatía con los alumnos, así como el apoyo psicosocial, cuando se hace necesario.

Al respecto, los tutores manifestaron que los estudiantes son muy distintos, pues mientras algunos esperan una guía muy puntual y abundantes indicaciones sobre lo que han de realizar como alumnos del Programa, hay otros que, incluso atravesando por situaciones difíciles y adversas, no se abren a solicitar apoyo u orientación.

... ¿qué pasa cuando no quiere que le des apoyo?... (p2, 93), refiriendo aspectos como la libertad y el respeto a su decisión de no querer hablar de su problema particular.

Lo que deja ver que los alumnos en su interacción con el tutor, marcan su distancia y el posible acercamiento; pues si bien la construcción del trabajo está de por medio en este vínculo, el clima de trabajo y de confianza que se establece, va dando la posibilidad de que los alumnos soliciten apoyo o consejo cuando lo necesitan, aspecto que llevó al tutor plantearse la contraparte de esta relación.

... ¿cuáles son las competencias de un alumno de posgrado? (para incorporarse a la tutoría).... falta la otra parte... (p4 g2).

Los tutores argumentaron que si bien se les asignan alumnos cuyos intereses son afines al campo de conocimientos que es de su dominio, algunos de ellos en un momento inicial no tienen claridad sobre lo que pretenden investigar, o bien su proyecto de investigación no es viable metodológicamente, por lo que requieren de mucha orientación.

En este contexto una tutora emitió el siguiente comentario:

...La tutoría es una actividad compleja... (p793) argumentando que es un encuentro entre dos personas adultas, donde se espera que prevalezca un interés genuino sobre lo que se hace y en donde se espera que el tutor tenga la capacidad de comprender al alumno.

\section{Profesionalismo del tutor y perfiles de los alumnos}

Al referirse a la competencia de profesionalismo del tutor, se mencionó que esta refiere el compromiso y responsabilidad que muestra el tutor en la formación del alumno, en este sentido los tutores manifestaron que para cubrir dicha competencia, interfiere el perfil socio-académico de los alumnos con el que se trabaja, como sucedió con la generación estudiada en donde se contó con la asistencia de estudiantes que provenían de algún estado del país, los cuales mostraron características y perfiles muy distintos, que en algunos casos demandaron una tutela más indicativa. 
...un perfil que es distinto de la población del centro, te puede provocar grandes dificultades con referentes socioculturales muy diferentes...se rompe la comunicación y estamos en otra frecuencia... (p1, g2).

...no puede ser que mi función de tutor termine como si fuera un hijo... aunque se equivoquen deben tener la experiencia... (p 5, g1).

En este caso se destacó la necesidad de favorecer la libertad e iniciativa del alumno versus la dependencia o la demanda de una acción muy directiva.

\section{DISCUSIÓN}

Al interior de los grupos focales se discutió a partir de la revisión y el análisis de las diez competencias que presentan los tutores de posgrado, contenidas en una rúbrica de autoevaluación, descritas en forma cualitativa en función de cuatro niveles de dominio: incipiente, en desarrollo, consumado y ejemplar ${ }^{18}$. El análisis se centró en las categorías de comportamiento ético del tutor; la transición de la investigación descriptiva a la intervención controlada; y la libertad del alumno en su relación con el tutor. Es decir, aspectos que se vinculan con las competencias de formación para la investigación y con las competencias interpersonales como es el comportamiento ético, el clima de interacción y el profesionalismo del tutor (Tabla 1); sin embargo, la competencia que alude al auspicio académico, no suscitó ni captó interés por parte de los tutores en ninguno de los tres grupos focales.

El elemento ético en la práctica educativa, es un aspecto que ha documentado ampliamente Ana Hirsh $^{25}$ en sus investigaciones, recupera información de los alumnos de diferentes posgrados, que coincide con lo expuesto en este caso y de manera especial con el tema del respeto a las autorías. Este aspecto también tiene una importante presencia en el seguimiento que han realizado los Comités de Ética de la Investigación y la Docencia de algunas instituciones educativas de nivel superior ${ }^{26}$ que velan por la integridad y la observancia en la práctica de los valores universitarios.

Al abordar la competencia de formación para la investigación, los tutores consideraron que las descripciones cualitativas contenidas en el instrumento de la rúbrica, son más afines a los posgrados de investigación, en los que se espera que las aportaciones de las investigaciones, se traduzcan en la generación de nuevos conocimientos, sin embargo en el caso de los posgrados profesionalizantes, cuyos resultados se orientan más a la solución de problemáticas muy específicas por parte del profesional. En este mismo sentido se pronuncia Sánchez-Mariñez ${ }^{27}$ y Medina-López ${ }^{28}$, quienes refieren que una aportación de las maestrías profesionalizantes son los estudios de intervención, la elaboración de prototipos, la elaboración de revisiones sistemáticas y críticas de estados del arte del ejercicio profesional o bien la elaboración de un estudio de la práctica que se apega a los estándares que establece la propia disciplina, aspectos que representan aportes a la investigación, pero desde la mirada de profesionales que enfrentan dificultades concretas en el campo de intervención del ejercicio profesional.

Los datos que emergieron relativos a la libertad del alumno en su relación con el tutor, mostraron la heterogeneidad de la población estudiantil y la diversidad de requerimientos que los tutores reciben. En este mismo sentido, se pronuncian los trabajos de Campbell y Campbell ${ }^{29}$ quienes consideran que las necesidades de los alumnos pueden aludir a una gama muy diversa de razones y destacaron que los tutorados tienden a vincularse con sus tutores fundamentalmente para recibir ayuda al tomar decisiones académicas, recibir consejos y guía académica sobre requisitos del grado o bien recibir apoyo en crisis personales. 
Las aportaciones de Difabio de Anglat ${ }^{6}$, destacan que si bien el trabajo del tutor del posgrado no se limita a la revisión de la tesis del alumno, ésta resulta más productiva si se realiza a manera de una retroalimentación constructiva del trabajo, mientras que los trabajos de Barrón³, Pérez y Arredondo ${ }^{15}$, así como Ducoing ${ }^{30}$, interpelan a la libertad del tutorado, siempre y cuando se trate de favorecer tanto su independencia intelectual como su formación integral, aspectos que también emergieron en los grupos focales.

En este sentido, los tutores de este estudio destacaron que la selección de los aspirantes, debe considerar dichos aspectos y que, si bien se habla de las competencias del tutor de posgrado, paralelamente deben considerarse las competencias de los alumnos del posgrado.

\section{CONCLUSIONES}

Esta investigación-acción tuvo como propósito que los tutores de un programa de maestría en enfermería, autoevalúen sus competencias como tutores, a la luz de una rúbrica que describe en forma cualitativa diez competencias de la tutoría en el posgrado, con cuatro niveles de dominio. La reflexión de su propia experiencia y práctica educativa, contribuyó a esclarecer lo que la institución educativa espera de su desempeño en este nivel y a su vez propició su autoevaluación tanto a nivel individual como grupal.

Al interior de los grupos focales, los tutores pudieron reconocer dichas competencias y valorar que tan lejos o cerca está cada uno de ellos(as) de tales referentes, destacando paralelamente aspectos que han de promoverse desde el contexto institucional del Programa, como es el proceso de selección de los aspirantes. Asimismo, los tutores consideraron que algunos rasgos de las competencias, no se apegan en una justa dimensión al nivel específico de los estudios de maestría en el que participan, como es la competencia de formación en investigación cuyo nivel superior de desarrollo (en la rúbrica) corresponde a un nivel de doctorado.

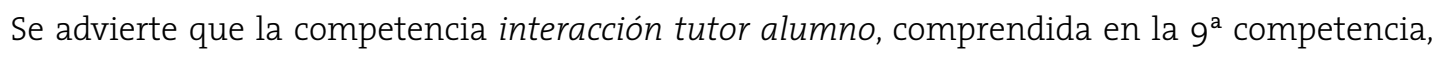
referente al clima de interacción, empatía, confianza y comunicación con el alumno, es un elemento transversal en las otras competencias desplegadas por el tutor, por lo que ésta tuvo una presencia reiterada al interior de la discusión en los grupos focales.

Actualmente los modelos educativos, colocan en el centro el aprendizaje del alumno, así como el desarrollo de su capacidad de autorregulación, que le permitan continuar aprendiendo a lo largo de la vida, y en este sentido los argumentos expuestos por los tutores, apuntaron al establecimiento de una relación que promueva la libertad y la independencia creciente del estudiante, que lo conduzcan a tomar decisiones, asumir compromisos, con el propósito de conferirle seguridad como profesional y sentido de dominio en el campo de estudio de la enfermería. La apreciación de esta competencia y su nivel de logro, se ve en buena medida comprometida cuando el aspirante que ingresa, adopta un papel dependiente y sin iniciativa.

La competencia ética fue la más aludida en los tres grupos, se observaron diversas formas de conductas éticas y no éticas, generalmente vinculadas con el respeto a las autorías en las publicaciones de artículos, que muestra la necesidad de dar un justo reconocimiento al trabajo que realiza el tutor en su campo de estudio y al alumno tutorado cuando funge como colaborador.

La transferencia del conocimiento como un elemento constitutivo de la formación en el programa de maestría, destacó la necesidad de apuntalar la formación de recursos en enfermería, que contribuyan a resolver problemáticas de salud y generar prácticas innovadoras para el cuidado así como de su gestión, como lo indica el Programa curricular. En este sentido cobra relevancia 
la realización de trabajos de intervenciones de enfermería, como productos derivados de su formación en el posgrado.

La competencia vinculada con la consejería y el auspicio académico, que se relacionan con la obtención de recursos humanos, materiales, financieros o de infraestructura, para realizar proyectos de investigación, no recibió atención por parte de los tutores de ninguno de los grupos focales; paralelamente reflejó la competencia con menor nivel de dominio en la autoevaluación que formularon de sí mismos.

La competencia de socialización que alude a la integración de los alumnos a los grupos profesionales o de investigación, también mostró un nivel de competencia incipiente, aspecto que guarda relación con la participación y consolidación de las líneas de investigación en las que se inscriben y producen los tutores del Programa.

Por último, los datos reflejan que la mayoría de los tutores participantes, cuenta con una larga trayectoria en el Programa, que les ha dado la oportunidad de interactuar con una gran diversidad de alumnos y confrontado diversas situaciones para guiarlos hasta la conclusión de su trayecto de formación, lo que les permite ubicarse a sí mismos preponderantemente en los niveles en desarrollo y consumado, de manera especial en el caso de la competencia de Docencia, que guía el proceso formativo de los alumnos y promueve una visión amplia del campo de conocimientos y de intervención de enfermería.

Una limitación del estudio es que, si bien se muestra la perspectiva de los tutores, queda ausente la visión de los tutorados, cuyas aportaciones sobre esta temática, mostraría una faceta sin duda de gran valor.

\section{RESPONSABILIDADES ÉTICAS}

Protección de personas o animales. Las autoras declaran que en esta investigación no se realizó ningún experimento con seres humanos.

Confidencialidad. Las autoras declaran que en este documento no aparecen los datos de los participantes, garantizando su anonimato y el manejo confidencial de la información.

Conflicto de intereses. Las autoras declaran no tener conflicto de intereses.

Financiamiento. Ninguno.

Agradecimientos. Agradecemos a los tutores su participación libre y voluntaria en los grupos de discusión, pues sin ella no habría sido posible la realización de este trabajo.

\section{REFERENCIAS}

1. De la Cruz-Flores G, Chehaybar-y Kury E, Abreu LF. Tutoría en educación superior: una revisión analítica de la literatura. Rev. educ. sup. 2011; 40(157): 189-209. https://bit.ly/2KFGo6N

2. López-Martín I, González- Villanueva P, Velasco-Quintana PJ. Ser y ejercer de tutor en la universidad. Red U. 2013; 11(2): 107-34. https://doi.org/10.4995/redu.2013.5569

3. Barrón C. Repensar la formación de los tutores. En: Ducoing P (Coord.). Tutoría y Mediación I. México, D.F.: Universidad Nacional Autónoma de México, Instituto de Investigaciones sobre la Universidad y la Educación; 2009. pp. 215-32. https://bit.ly/33uTmcM

4. De la Cruz-Flores G, Abreu-Hernández LF. Atributos de tutores del posgrado por campo disciplinario. La perspectiva de estudiantes de la Universidad Nacional Autónoma de México. Perf. educ. 2012; 34(138): 10-27. https://doi.org/10.22201/iisue.24486167e.2012.138.34152 
5. Martínez-González A, Laguna-Calderón J, García-Sahagún MC, Vázquez-Padilla MI, RodríguezCarranza R. Perfil de competencias del tutor de posgrado de la Universidad Nacional Autónoma de México. México, D.F.: Universidad Nacional Autónoma de México; 2005.

6. Difabio-de Anglat $H$. Las funciones del tutor de la tesis doctoral en educación. Rev. Mex. Inv. Educ. 2011; 16(50): 935-59. https://bit.ly/2KVAWLN

7. González-Juárez G. Tutoría y competencias de investigación en los alumnos de posgrado de la UNAM. México, D.F.: Ediciones Díaz de Santos / Universidad Nacional Autónoma de México; 2015.

8. De la Cruz G, Abreu LF. Tutoría en Posgrado: agente mediador en la formación de guardianes de la disciplina. En: Ducoing P (Coord.). Tutoría y Mediación I. México, D.F.: Universidad Nacional Autónoma de México, Instituto de Investigaciones sobre la Universidad y la Educación; 2009. pp. 191-208. https://bit.ly/33uTmcM

9. Moreno T. El profesor-tutor universitario en el contexto de la sociedad del conocimiento. En: Ducoing P (Coord.).Tutoría y Mediación I. México, D.F.: Universidad Nacional Autónoma de México, Instituto de Investigaciones sobre la Universidad y la Educación; 2009. pp. 233-56. https://bit.ly/33uTmcM

10. Cardoso-Espinosa EO, Cerecedo-Mercado MT, Ramos-Mendoza JR. Autoevaluación de las competencias docentes en los Posgrados de Administración del Instituto Politécnico Nacional. Rev. estud. exp. educ. 2014; 13(25): 33-47. https://bit.ly/3mopogh

11. Rueda-Beltrán M (Coord.). ¿Evaluar para controlar o para mejorar? Valoración del desempeño docente en las universidades. México, D.F.: Universidad Nacional Autónoma de México, Instituto de Investigaciones sobre la Universidad y la Educación; 2011.

12. De Diego-Correa $M$, Rueda-Beltrán $M$. La evaluación docente en educación superior: uso de instrumentos de autoevaluación, planeación y evaluación de pares. Voces silec. rev. latinoam. educ. 2012; 3(2): 59-76. https://doi.org/10.18175/vys3.2.2012.04

13. Hernández CA, Jiménez M, Guadarrama E, Rivera AE. La percepción de la motivación y satisfacción de la tutoría recibida en estudios de posgrado. Form. Univ. 2016; 9(2): 49-58.

http://dx.doi.org/10.4067/So718-50062016000200006

14. Morán-Peña L, Ostiguín-Meléndez RM. Ser tutor en el Posgrado de Enfermería. México: Universidad Nacional Autónoma de México, Escuela Nacional de Enfermería y Obstetricia; 2005.

15. Pérez G, Arredondo M. La tutoría en algunos posgrados de la Universidad Nacional Autónoma de México. En: Ducoing P (Coord.). Tutoría y Mediación I. México, D.F.: Universidad Nacional Autónoma de México, Instituto de Investigaciones sobre la Universidad y la Educación; 2009. pp. 149-74. https://bit.ly/33uTmcM

16. Perrenoud P. Diez competencias para enseñar. $5^{a}$ reimp. Barcelona: Graó; 2008.

17. Denyer M, Furnémont J, Poulain R, Vanloubbeeck G. Las competencias en educación: Un balance. México: Fondo de Cultura Económica; 2007.

18. De la Cruz-Flores G, Díaz-Barriga Arceo F, Abreu-Hernández LF. La labor tutorial en los estudios de posgrado: Rúbricas para guiar su desempeño y evaluación. Perf. educ. 2010; 32(130): 83-102.

https://doi.org/10.22201/iisue.24486167e.2010.130.20624

19. Smitter Y. Lineamientos para la autoevaluación del desempeño docente en las funciones de docencia, investigación y extensión en el Instituto Pedagógico de Miranda José Manuel Siso Martínez. Inves. Post. 2008; 23(3): 281-98. https://bit.ly/37Ga1LR

20. Schön DA. La formación de profesionales reflexivos: Hacia un nuevo diseño de la enseñanza y el aprendizaje en las profesiones. Barcelona: Paidós; 1992. 
21. Perrenoud P. Desarrollar la práctica reflexiva en el oficio de enseñar: Profesionalización y razón pedagógica. $1^{\text {ra }}$ ed (4 ${ }^{\text {ta }}$ reimpr.). Barcelona: Graó/ Colofón; 2011.

22. San Fabián-Maroto JL, Granda-Cabrales A. Autoevaluación de centros educativos. Madrid: Síntesis; 2013.

23. Santos-Guerra MA. La evaluación como aprendizaje: una flecha en la diana. 1 $^{\mathrm{a}}$ ed. Buenos Aires: Bonum; 2007.

24. Carr W, Kemmis, S. Teoría crítica de la enseñanza: La investigación-acción en la formación del profesorado. Barcelona: Martínez Roca; 1988.

25. Hirsch-Adler A. Competencias y rasgos de ética profesional en estudiantes y profesores de posgrado de la UNAM. Sinéctica. 2009; (32): 1-16. https://bit.ly/3lAvomE

26. Graue-Wiechers EL. Acuerdo por el que se establecen los lineamientos para la integración, conformación y registro de los comités de ética en la Universidad Nacional Autónoma de México. Gaceta UNAM. 2019 ago 29; Secc. Acuerdos y convocatorias. pp. 21-26. https://bit.ly/39GofPg

27. Sánchez-Marínez J. Una propuesta conceptual para diferenciar los programas de postgrado profesionalizantes y orientados a la investigación: Implicaciones para la regulación, el diseño y la implementación de estudios de postgrado. Cienc. Soc. 2008; 33(3): 327-41. https://bit.ly/2JNCiYA

28. Medina-López ER. La investigación en los posgrados académicos y profesionalizantes en Honduras. Paradigma. 2015; 21(35): 33-52. https://doi.org/10.5377/paradigma.v21i35.2275

29. Campbell D, Campbell T. The mentoring relationships: differing perceptions of benefits. Coll Stud J. 2000; 34(4): 516-23. https://bit.ly/33Epafc

30. Ducoing P. ¿Tutoría y/o acompañamiento en educación?. En: Ducoing P (Coord.). Tutoría y Mediación I. México, D.F.: Universidad Nacional Autónoma de México, Instituto de Investigaciones sobre la Universidad y la Educación; 2009. pp. 57-80. https://bit.ly/33uTmcM 